\title{
Kernos
}

Revue internationale et pluridisciplinaire de religion grecque antique

30 | 2017

Varia

\section{Private Associations and the Public Sphere}

\section{Romain Guicharrousse}

\section{OpenEdition \\ Journals}

\section{Édition électronique}

URL : http://journals.openedition.org/kernos/2541

DOI : 10.4000/kernos.2541

ISSN : 2034-7871

\section{Éditeur}

Centre international d'étude de la religion grecque antique

\section{Édition imprimée}

Date de publication : 1 octobre 2017

Pagination : 352-354

ISSN : 0776-3824

\section{Référence électronique}

Romain Guicharrousse, "Private Associations and the Public Sphere », Kernos [En ligne], 30 | 2017, mis en ligne le 01 octobre 2017, consulté le 24 septembre 2020. URL : http://journals.openedition.org/ kernos/2541; DOI : https://doi.org/10.4000/kernos.2541

Ce document a été généré automatiquement le 24 septembre 2020.

Kernos 


\title{
Private Associations and the Public Sphere
}

\author{
Romain Guicharrousse
}

\section{RÉFÉRENCE}

Vincent GABRIELSEN, Christian A. THOMSEN (éd.), Private Associations and the Public Sphere: Proceedings of a Symposium held at the Royal Danish Academy of Sciences and Letters, 9-11 September 2010, Copenhague, The Royal Danish Academy of Sciences and Letters, 2015. 1 vol. 14,9 × 22,9 cm, 362 p. (Scientia Danica. Series H, Humanistic 8, vol. 9). ISBN :978-87-7304-389-9.

1 Première pierre du projet CAP (Copenhagen Associations Project) de l'Université de Copenhague, ce volume est issu d'un colloque tenu à l'Académie royale des Sciences et Lettres du Danemark en 2010. Réunissant onze communications, il offre au lecteur un panorama bienvenu de la variété des associations privées dans les mondes grec et romain $d u v^{e}$ siècle avant notre ère au III $^{\mathrm{e}}$ siècle de notre ère, et de leur rapport avec les autorités civiques ou royales en place en Grèce égéenne, en Asie mineure et dans l'Égypte grecque et romaine.

Dans une solide introduction, les deux éditeurs invitent à définir l'association privée, cœur du projet CAP, comme une structure possédant un nom qui lui est propre, une organisation et une existence durable - ou qui se veut durable à l'origine - , et des règles d'adhésion pour être membre. Le but du volume est de confronter ces associations privées à la sphère publique, dont la délimitation est, de l'aveu des auteurs, complexe. Bien que soulignant les limites d'une telle définition, ceux-ci l'identifient à la sphère étatique, c'est-à-dire les domaines qui sont du ressort de la cité ou du roi. Ils souhaitent dès lors interroger les liens qui pourraient exister entre des associations privées, phénomène au développement considérable dans l'Antiquité, et l'État.

Chaque contribution prend le temps de bien contextualiser son objet d'étude dans le temps comme dans l'espace - effort louable, tant les situations sont variées. Chaque 
association est également présentée et sa structure interne décrite. On remarque rapidement que, comme le soulignent la plupart des auteurs, ces associations sont structurées sur le modèle de la cité grecque, que l'on se trouve dans une association religieuse athénienne en l'honneur d'une divinité étrangère (Bendis, chapitre 2) ou dans une communauté chrétienne pastorale $\mathrm{du} \mathrm{I}^{\text {er }}$ siècle de notre ère en Crète (chapitre 8). Chaque association possède des magistrats, des critères d'adhésion précis. Seule exception, les associations de marchands d'Égypte ptolémaïque dont le modèle est celui du nome ou du village (chapitre 9).

4 Malgré ces structures similaires, ces associations possèdent des dénominations très variées, dont les spécificités sont parfois difficiles à affirmer. Si l'étude, au chapitre 11, du monde ptolémaïque permet de faire la part entre un ethnos (groupe fondé sur la taxation) et un politeuma (groupe essentiellement militaire), le terme générique koinon, fréquent dans tout le monde grec, regroupe des associations aux desseins bien différents. Les raisons d'être de ces koina du monde grec et égyptien dans l'Antiquité sont, en effet, très variées. On retrouve ainsi des associations religieuses (les associations de Bendis à Athènes, chapitre 1; les associations pastorales en Asie mineure et en Crète, chapitre 8), de très nombreuses associations professionnelles (associations de médecins et de professeurs en Asie mineure, chapitre 4 ; association de boulangers à Éphèse, chapitre 7; associations de marchands en Égypte, chapitre 9), et des associations fondées sur des critères variés, comme l'origine ethnique ou le statut juridique (les étrangers à Rhodes, chapitre 6). Dans la plupart des cas cependant, ces associations ne se limitaient pas à un domaine en particulier : ainsi les associations juives de militaires en Égypte ptolémaïque possèdent une double composante religieuse et professionnelle (chapitres 10 et 11) et les apiculteurs du nome d'Arsinoite sont organisés autour d'un temple (p. 309) où se déploient leurs activités communes.

5 Si les associations avaient un but premier, comme la défense des intérêts des professionnels (ainsi les médecins et professeurs d'Asie mineure, que M.P. de Hoz compare aux technites dionysiaques bien connus pour la période hellénistique, p. 103), leurs activités se déployaient dans des domaines bien plus variés. Ces associations privées possédaient des fonctions sociales importantes pour leurs membres : ainsi les écoles philosophiques athéniennes ne manquaient pas d'organiser des banquets (chapitre 3), les associations de médecins et professeurs avaient la charge de préserver et d'entretenir les tombes de leurs anciens membres (chapitre 4, p. 110), etc. Plusieurs contributions soulignent ainsi le puissant ferment social que représentent ces groupes privés. Pour certaines, elles servaient la mixité sociale, en incluant des femmes, des esclaves (association d'étrangers à Rhodes, chapitre 6, p. 144), mais elles permettaient aussi de préserver l'ordre social, dont elles prêchaient le respect: les associations pastorales chrétiennes invitaient leurs membres à la soumission aux autorités civiles, en vue de préserver la concorde dans les cités (chapitre 8). Elles constituaient un moyen pour certains notables d'asseoir et de conserver leur place dans la cité. Dans le cas des étrangers rhodiens, analysés par Stéphanie Maillot, les magistrats des associations prennent ainsi une part active dans la vie de la cité, au contact des élites locales.

Ces contacts montrent que les associations sont loin d'être refermées sur elles-mêmes, et sont fréquemment en relation avec les cités ou les administrations royales. L'intérêt central de l'ouvrage se situe dans l'exposition des multiples modes d'être de ces groupes à l'autorité étatique. Trois perspectives peuvent être dégagées de l'ensemble 
des contributions. Tout d'abord, d'une manière large, certaines associations sont étudiées au sein de l'espace public. Ainsi les écoles philosophiques, analysées par Matthias Haake, sont reçues diversement à Athènes : si elles sont moquées sur la scène du théâtre, notamment au $v^{e}$ siècle par Aristophane, elles finissent par être reconnues, à tel point que l'éducation des éphèbes est confiée aux maîtres à partir des années 120 avant notre ère.

7 Ces groupes associatifs constituaient des objets de discussion dans la cité : les citoyens athéniens réunis en assemblée ont ainsi débattu, à la fin du ve siècle, de la place des orgéons de la déesse Bendis dans la cité (chapitre 2). Réalisant des sacrifices au nom de la cité, l'Assemblée s'est interrogée ainsi sur la question de la prêtrise de ce culte nouvellement introduit. Ils ont, par ailleurs, délibéré à la fin du $\mathrm{IV}^{\mathrm{e}}$ siècle sur le bienfondé de l'existence à Athènes des écoles philosophiques (loi de Sophoclès, chapitre 3). Les associations étaient donc un sujet de débats dans les cités grecques et dans les royaumes hellénistiques.

8 L'ensemble des communications de cet ouvrage montre clairement que les rapports entre ces associations privées et les autorités civiles s'étendaient au sein d'un large spectre qui allait du pur conflit et de l'interdiction (les écoles philosophiques à la fin du IV siècle, chapitre 3 ; la "grève " des boulangers d'Éphèse, chapitre 7) à la pleine coopération. Celle-ci pouvait se manifester par le don de privilèges en échange de contributions financières à des souscriptions publiques (Rhodes, chapitre 6), comme par le fait de se voir confier d'importantes prérogatives : les Juifs d'Héracléopolis en Égypte au II $^{\mathrm{e}}$ siècle avant notre ère, en échange d'un plein soutien militaire, possèdent un rôle administratif formel, dont l'arbitrage en cas de conflit juridique entre les membres de l'association, mais aussi en dehors. Cette coopération n'est cependant pas toujours évidente : dans le cas des associations professionnelles en Égypte à la période romaine, des conflits légaux peuvent survenir entre les officiels du pouvoir impérial et des associations qui se sont vues confier l'organisation du travail dans certaines branches. Ces conflits nécessitent l'intervention de l'administration provinciale, parfois en faveur des associations (chapitre 12, p. 328), signe que les associations sont des interlocuteurs reconnus et ont un poids politique dans certaines cités.

9 La variété des interactions entre les "associations privées » et la "sphère publique " invite finalement à s'interroger sur la pertinence heuristique d'une telle séparation que l'historiographie française a remise en cause depuis longtemps ${ }^{1}$. En effet, ce qui ressort de l'ensemble des communications est l'entrecroisement permanent des deux sphères d'étude, comme l'avoue d'ailleurs M.P. de Hoz: «from the moment an association of teachers and physicians was founded, the private/public opposition nolonger seemed to have any more significance for the association and its representation in the community 》(p. 118119). Si les deux éditeurs semblent avoir conscience de cet enchevêtrement permanent (p. 12-14), ils s'en tiennent pourtant à cette opposition discutable.

10 Malgré ce bémol, et les quelques coquilles qui parsèment certains chapitres, cet ouvrage ouvre, par la variété des phénomènes associatifs répertoriés, bien des perspectives dans un domaine de la recherche historique dynamique et permet de faire le point à la fois sur la structure de nombreux types d'associations, et de montrer la multiplicité des rapports avec les autorités civiques ou royales. Les onze contributions, bien que centrées sur le rapport public/privé, suggèrent de nombreuses pistes d'analyse du phénomène associatif dans le monde grec de l'époque archaïque à l'époque impériale. On sait donc gré au prometteur Copenhagen Associations Project de 
nous offrir ce premier volume issu de leurs recherches et de permettre la comparaison spatio-temporelle d'un phénomène juridique et social infra-civique qui touche toutes les parties du monde grec durant toute l'Antiquité.

\section{NOTES}

1. Voir notamment P. SCHMitT PANTEL, F. DE POLIGNAC, Public et privé en Grèce ancienne: lieux, conduites, pratiques. Actes du colloque organisé à Paris les 15-17 mars 1995, Ktèma 23 (1998).

\section{AUTEURS}

\section{ROMAIN GUICHARROUSSE}

Université Paris 1 Panthéon Sorbonne / École Française d'Athènes 